\title{
Bile acid patterns in meconium are influenced by cholestasis of pregnancy and not altered by ursodeoxycholic acid treatment
}

\author{
C M P Rodrigues, J J G Marín, D Brites
}

\begin{abstract}
Background-Data on meconium bile acid composition in newborn babies of patients with intrahepatic cholestasis of pregnancy (ICP) are relatively scant, and changes that occur on ursodeoxycholic acid (UDCA) administration have not been evaluated.
\end{abstract}

Aims-To investigate bile acid profiles in meconium of neonates from untreated and UDCA treated patients with ICP. Maternal serum bile acid composition was also analysed both at diagnosis and delivery to determine whether this influences the concentration and proportion of bile acids in the meconium.

Patients/Methods-The population included eight healthy pregnant women and 16 patients with ICP, nine of which received UDCA $(12.5-15.0 \mathrm{mg} / \mathrm{kg}$ body weight/day) for $15 \pm 4$ days until parturition. Bile acids were assessed in the meconium by gas chromatography-mass spectrometry and in maternal serum by high performance liquid chromatography. Results-Total bile acid and cholic acid concentrations in the meconium were increased $(p<0.01)$ in newborns from patients with ICP (13.5 (5.1) and 8.4 (4.1) $\mu \mathrm{mol} / \mathrm{g}$ respectively; mean (SEM)) as compared with controls $(2.0(0.5)$ and 0.8 (0.3) $\mu \mathrm{mol} / \mathrm{g}$ respectively), reflecting the total bile acid and cholic acid levels in the maternal serum $(r=0.85$ and $r=0.84$, p<0.01). After UDCA administration, total bile acid concentrations decreased in the mother $(\sim 3$-fold, $p<0.05)$ but not in the meconium. UDCA concentration in the meconium showed only a 2 -fold increase after treatment, despite the much greater increase in the maternal serum $(p<0.01)$. Lithocholic acid concentration in the meconium was not increased by UDCA treatment.

Conclusions-UDCA administration does not influence the concentration and proportion of bile acids in the meconium, which in turn are altered by ICP. Moreover, this beneficial treatment for the mother does not increase meconium levels of potentially toxic metabolites of UDCA such as lithocholic acid.

(Gut 1999;45:446-452)

Keywords: bile acids; cholestasis; pregnancy; cholic acid; meconium; ursodeoxycholic acid therapy
Intrahepatic cholestasis of pregnancy (ICP) is generally considered benign to the mother although it often causes pruritus and biochemical cholestasis. ${ }^{1-3}$ Increased fetal distress, premature deliveries, and perinatal mortality and morbidity have been described ${ }^{4-6}$ and raised some concerns. Moreover, high frequency of meconium staining of the amniotic fluid may be considered a sign of poor prognosis for the fetus. ${ }^{5}$ It may be possible that harmful effects on fetal wellbeing result from high bile acid levels in amniotic fluid and serum cord blood, reflecting an appreciable increase in bile acids in maternal serum, ${ }^{8-11}$ as well as from a reduced ability of the fetus to eliminate bile acids across the placenta. ${ }^{12}$

Ursodeoxycholic acid (UDCA) administration during ICP has been shown to improve both pruritus and liver function tests, correct maternal serum bile acid profile, ${ }^{13-21}$ and decrease passage of bile acids to the fetus. ${ }^{22} 23$ Furthermore, a valuable contribution to favourable fetal outcome is the ability of UDCA to reverse the impairment of bile acid transport across the trophoblast. ${ }^{12}$

An investigation of the bile acid pattern in meconium of newborn babies of patients with ICP may give some insight into whether cholestasis in the mother is a governing factor in meconium bile acid composition. Meconium comprises a concentrate of metabolites of both fetal and maternal origin, in which the bile acid composition is influenced by the presence of bile in the fetal duodenum and by the ingestion of amniotic fluid. Moreover, equilibrium between the fetal bile acid pool and the intestinal contents appears to be restricted because of the poor fetal enterohepatic circulation of bile acids. The occurrence of a pattern of unusual bile acids in the meconium, similar to that of patients with cholestatic liver disease, has been attributed to physiological cholestasis during the developmental phase. ${ }^{24-28}$ It is therefore tempting to speculate that UDCA may have some beneficial effects on the cholestatic pattern of bile acids in the meconium during ICP.

\section{Methods}

STUDY PROTOCOL

This study was conducted in collaboration with Santa Maria Hospital, Garcia de Orta Hospital, and Dr Alfredo da Costa Maternity Ward from 1994 to 1997. Eight healthy volunteers at

Abbreviations used in this paper: ICP, intrahepatic cholestasis of pregnancy; UDCA, ursodeoxycholic acid; GC-MS, gas chromatography-mass spectrometry.
Accepted for publication 3 March 1999 
full term gestation were enrolled and considered as the control group. None had a history of hepatic disease or pre-eclampsia. Sixteen patients with ICP were included in the study. Inclusion criteria were as previously described $^{420}$ : (a) severe pruritus; (b) total bile acids above $11 \mu \mathrm{mol} / 1$, cholic acid percentage higher than $40 \%$, and increased alanine aminotransferase or conjugated bilirubin; (c) absence of any other known disease that could cause liver malfunction. According to the severity of the ICP disease, seven patients were given classical palliative treatments (less severe, "non-UDCA group"), and nine received UDCA (more severe, "UDCA group") at $12.5-15.0 \mathrm{mg} / \mathrm{kg}$ body weight/day beginning 20 days or less ( $15 \pm 4$ days, 9-20 days) before term. Pruritus assessment and its changes during UDCA treatment were based on complaints from the patients. Four women in the UDCA group had previously suffered from cholestasis of pregnancy. The drug was discontinued at delivery and fasting maternal serum as well as meconium samples were collected. Meconium and maternal serum samples were also collected from eight uncomplicated pregnancies at term. Specimens were stored under a nitrogen environment at $-20^{\circ} \mathrm{C}$, after flashfreezing in liquid nitrogen, until analysed.

Informed consent was obtained from each patient and the study protocol conformed with the ethical guidelines of the 1975 Declaration of Helsinki.

\section{ANALYTICAL TECHNIQUES \\ Liver function tests}

Routine laboratory methods were used to determine serum biochemical markers of cholestasis including aspartate aminotransferase, alanine aminotransferase, $\gamma$-glutamyltranspeptidase, alkaline phosphatase, and total and conjugated bilirubin.

\section{Bile acid analysis in maternal serum}

Bile acids were measured in maternal serum, collected at both diagnosis and delivery, by high performance liquid chromatography and an enzymic-fluorimetric assay.

Bile acid conjugates - Individual amidated bile acids were determined by high performance liquid chromatography, essentially as described by Labbé et $a l^{2}$ with minor modifications. Briefly, after liquid-solid extraction using SepPak $\mathrm{C}_{18}$ cartridges (Waters Corp, Milford, Massachusetts, USA), ${ }^{30}$ separation of the principal glycine and taurine conjugated species in the maternal serum was achieved using a Shimadzu liquid chromatograph system, equipped with a variable wavelength ultraviolet detector and housing a reverse-phase LiChrospher 100 RP-8 endcapped column (Merck, Darmstadt, Germany).

Unconjugated bile acids-These species were evaluated using an enzymic-fluorimetric method, ${ }^{3132}$ after solid-phase extraction and thin layer chromatography using silica gel plates. ${ }^{33-35}$ Unconjugated UDCA in serum was not individually quantified because of the diffi- culty of separating it from chenodeoxycholic acid during thin layer chromatography.

Bile acid analysis in meconium-Bile acids were assessed in meconium by gas chromatographymass spectrometry (GC-MS) after liquid-solid extraction, hydrolysis, isolation by lipophilic anion exchange chromatography, and conversion to methyl ester-trimethylsilyl ether derivatives.

Total and individual bile acids-Meconium samples were weighed (0.1-0.5 mg), and $80 \%$ methanol $(50 \mathrm{ml})$ was added. All samples were sonicated for 30 minutes, refluxed for two hours, and then cooled and filtered. ${ }^{36}$ The residue was resuspended in $50 \mathrm{ml}$ chloroform/ methanol $(1: 1, \mathrm{v} / \mathrm{v})$, refluxed for one hour, and filtered. The combined extracts were taken to dryness under $\mathrm{N}_{2}$ and the dried extract was resuspended in $80 \%$ methanol. Exactly one fifth of the extract was taken, and the internal standard, nordeoxycholic acid (5 or $10 \mu \mathrm{g}$ ), was added. The sample was diluted with distilled water and passed through a Sep-Pak $\mathrm{C}_{18}$ cartridge. Bile acids were recovered by elution with methanol, and hydrolysis of conjugated species was performed as previously described. ${ }^{37} 38$ In short, solvolysis was carried out in a mixture of methanol, distilled tetrahydrofuran, and $1 \mathrm{M}$ trifluoroacetic acid in dioxane, heated to $45^{\circ} \mathrm{C}$ for two hours. Enzymic hydrolysis was achieved by overnight incubation with choloylglycine hydrolase (Sigma Chemical Co, St Louis, Missouri, USA) at $37^{\circ} \mathrm{C}$. After hydrolysis, bile acids were extracted by passage of the sample through a Sep-Pak column and recovered by elution with methanol. The unconjugated species, after hydrolysis, were isolated and separated from neutral sterols by anion exchange chromatography on diethylaminohydroxypropylSephadex LH-20 ${ }^{39}$ (Lipidex-DEAP; Packard Instrument Co, Groningen, The Netherlands). Recovery was achieved by elution with $0.1 \mathrm{M}$ acetic acid in $72 \%$ ethanol $(7 \mathrm{ml})$ and this fraction was evaporated to dryness. Methyl ester-trimethylsilyl ether derivatives were prepared $^{40}{ }^{41}$ and purified ${ }^{42}$ before separation by GC. GC-MS analysis was carried out using an HP 5890 series II gas chromatograph connected to an HP 5972 mass spectrometer. Identification of bile acids was based on the GC retention index relative to a homologous series of $n$-alkalis and the mass spectrum compared with authentic standards. When these were not available, identification was tentatively based on the predicted fragmentation patterns for specific bile acid structures. ${ }^{43}$ Quantification of bile acids was achieved using GC, by comparing the peak height response of the individual bile acids with that obtained from the internal standard.

Bile acid conjugates - Six of the meconium samples collected from newborns of patients with ICP treated or not with UDCA (three from each group, including a low, medium, and high total bile concentration sample) were analysed 
Table 1 Clinical data on patients with intrahepatic cholestasis of pregnancy

\begin{tabular}{lll}
\hline & Non-UDCA & UDCA \\
\hline Age (years) & $32.4(4.2)(26-36)$ & $30.8(4.8)(23-35)$ \\
Recurrence & 0 & 4 \\
Gemelar pregnancy & 1 & 0 \\
Onset of pruritus (weeks) & $33.1(3.2)(28-35)$ & $28.7(4.0)(26-34)^{\star}$ \\
Gestational age at diagnosis (weeks) & $35.4(1.8)(33-37)$ & $32.0(2.1)(29-35)^{\star \star}$ \\
Gestational age at delivery (weeks) & $36.5(2.2)(34-39)$ & $36.7(0.4)(35-39)$ \\
Green amniotic fluid & 2 & 3 \\
Newborns & $2870(660)(2110-3670)$ & $2902(232)(2490-3380)$ \\
$\quad$ Birth weight (g) & & \\
$\quad$ Apgar score & $8.6(0.9)$ & $9.0(0.0)$ \\
$\quad$ 1 minute & $9.8(0.5)$ & $10.0(0.0)$ \\
\hline
\end{tabular}

Patients with intrahepatic cholestasis of pregnancy received classical palliative therapy (non-UDCA group; $\mathrm{n}=7$ ) or were treated with ursodeoxycholic acid (UDCA) until labour (UDCA group; $n=9$ ). Values are expressed as mean $(\mathrm{SD})$ (respective range).

${ }^{\star} \mathrm{p}<0.05$ and ${ }^{\star \star} \mathrm{p}<0.01, v$ non-UDCA.

to evaluate the extent of bile acid conjugation. In addition, unconjugated and conjugated species were studied in three meconium samples from healthy newborns delivered from uncomplicated pregnancies. Methanolic extracts diluted to $72 \%$ methanol were passed through a column of Lipidex-DEAP, and unconjugated bile acids and their conjugates (glycine, taurine, and sulphate) were recovered by stepwise elution of the gel bed with appropriate buffers. ${ }^{44}$ After evaporation, conjugated species were hydrolysed, recovered, and converted to methyl ester-trimethylsilyl ether derivatives as described above.

\section{STATISTICAL ANALYSIS}

Data were compared using the Wilcoxon nonparametric rank sum test or the unpaired twotailed Student's $t$ test performed on the basis of equal or unequal variance as appropriate. Results are expressed as mean (SEM) or mean (SD), as indicated. The relation between maternal serum and meconium bile acid composition was examined by linear regression analysis. $\mathrm{p}<0.05$ was considered statistically significant.

\section{Results}

CLINICAL OBSERVATIONS

Statistical differences were noticed with respect to the onset of pruritus (33.1 (3.2) v 28.7 (4.0) weeks of gestation, $\mathrm{p}<0.05)$ as well as to the gestational age at diagnosis $(35.4$ (1.8) $v 32.0$ (2.1) weeks, $\mathrm{p}<0.01$ ) between patients with ICP receiving classic palliative treatment (non-
UDCA group) and those treated with UDCA (UDCA group) (table 1). The poor prognosis associated with an earlier onset of the disease led to the decision to start UDCA treatment in these patients and therefore justifies the statistical differences between the groups. No major side effects were reported in the nine patients studied during UDCA treatment. Compliance was assessed from the increased percentage of UDCA in the serum (higher than 18\%), and no drop outs were recorded during the treatment period. Itching disappeared or was appreciably reduced during UDCA treatment. All newborns were healthy and no differences were noticed for birth weight and Apgar scores when neonates from women included in the UDCA and non-UDCA groups were compared, suggesting a potential benefit of UDCA administration. Pruritus vanished and laboratory tests became normal within one and two weeks of labour respectively. Table 1 gives other clinical observations.

Patients with ICP showed significant increases $(p<0.01)$ in serum total bile acid concentrations (33.3 (11.4) v $5.0(0.3) \mu \mathrm{mol} / 1$ in control) and cholic acid percentage (61.3 (4.9) $v 35.2(1.1) \%$ ), as well as in alanine aminotransferase activity (324 (155) $v 24$ (4) $\mathrm{IU} / 1)$ and conjugated bilirubin levels (0.42 $(0.12) v 0.13(0.01) \mathrm{mg} / \mathrm{dl})$. In addition, $\gamma$-glutamyltranspeptidase activity was elevated in patients with ICP (34 (10) v 9 (1) IU/1, $\mathrm{p}<0.05)$. The group treated with UDCA showed significantly $(\mathrm{p}<0.05)$ lower serum total bile acid concentrations (13.0 (2.2) $\mu \mathrm{mol} /$ 1), cholic acid percentage (23.3 (3.4)\%), alanine aminotransferase (49 (23) IU/1), and $\gamma$-glutamyltranspeptidase activity (8 (1) IU/l), but not conjugated bilirubin concentration (0.39 (0.13) mg/dl, NS).

\section{BILE ACID ANALYSIS}

High total bile acid concentrations were found in meconium from newborns of the nonUDCA group when compared with the control group of uncomplicated pregnancies (13.5 (5.1) $v 2.0(0.5) \mu \mathrm{mol} / \mathrm{g}, \mathrm{p}<0.01)$, reflecting the elevated total bile acid concentrations in the maternal serum (figs 1 and 2). Reinforcing the influence of maternal serum bile acid composition on meconium bile acid profiles during ICP, cholic acid concentration was considerably
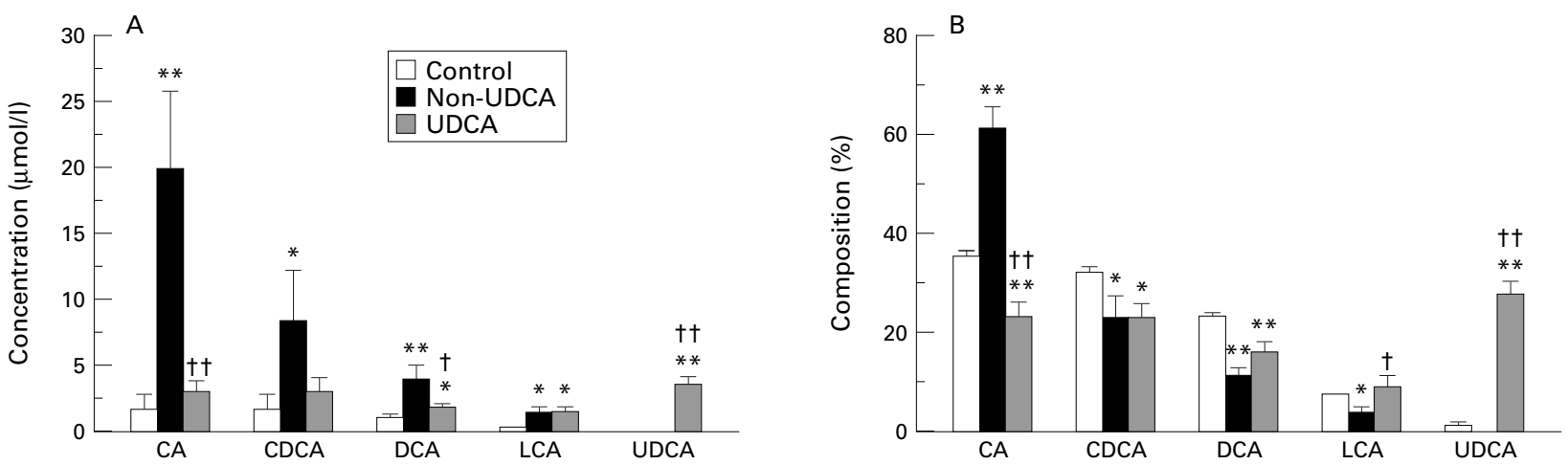

Figure 1 Concentration (A) and percentage composition (B) of the usual bile acids in serum of normal pregnant women (control; $n=8$ ) and patients with cholestasis of pregnancy receiving classical palliative treatment (non-UDCA group; $n=7$ ) or treated with ursodeoxycholic acid (UDCA) until labour (UDCA group; $n=9)$. Results are expressed as mean (SEM). ** $p<0.01$ and ${ }^{*} p<0.05$, non-UDCA and UDCA v control;ttp<0.01 and $t p<0.05$, non-UDCA vUDCA. CA, cholic acid; $C D C A$, chenodeoxycholic acid; $D C A$, deoxycholic acid; $L C A$, lithocholic acid. 

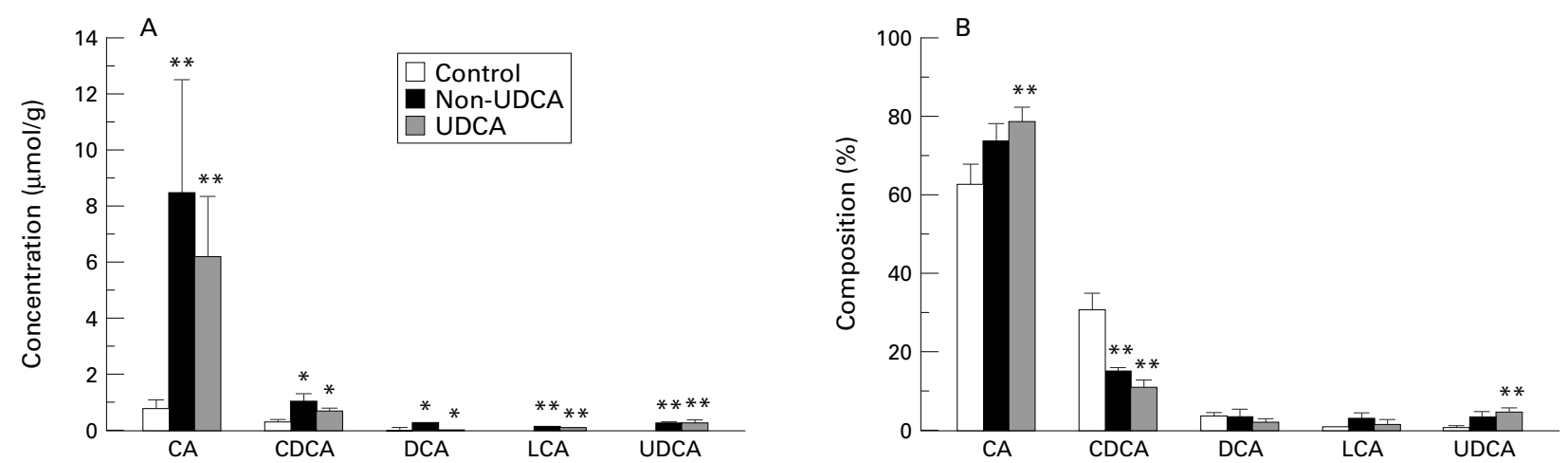

Figure 2 Concentration $(A)$ and percentage composition (B) of the usual bile acids in meconium samples of newborns from normal pregnancies (control; $n=8)$ and from pregnancies complicated by intrahepatic cholestasis of pregnancy receiving classical palliative treatment (non-UDCA group; $n=7)$ or treated with ursodeoxycholic acid (UDCA) until labour (UDCA group; $n=9$ ). Results are expressed as mean $(S E M)$. ${ }^{\star} p<0.01$ and ${ }^{\star} p<0.05$, non-UDCA and UDCA $v$ control. $C A$, cholic acid; $C D C A$, chenodeoxycholic acid; DCA, deoxycholic acid; LCA, lithocholic acid.

increased in both the maternal serum $(19.8$ (6.2) $v 1.7(0.1) \mu \mathrm{mol} / 1 ; \mathrm{p}<0.01)$ and the meconium (8.4 (4.1) $v 0.8 \quad(0.3) \quad \mu \mathrm{mol} / \mathrm{g}$; $\mathrm{p}<0.01)$. Even though this study was conducted using a limited population, there appears to be a relation between the concentration of total bile acids $(r=0.85 ; \mathrm{p}<0.01)$ and cholic acid $(r=0.84 ; \mathrm{p}<0.01)$ in maternal serum and meconium in ICP, which was not found in the controls. Surprisingly, after UDCA administration, total bile acid and cholic acid concentrations in the meconium (9.2 (2.2) and $6.1(2.2) \mu \mathrm{mol} / \mathrm{g}$ respectively) were no longer related to those in the maternal serum (17.2 (3.4) and 6.8 (2.6) $\mu \mathrm{mol} / \mathrm{l})$. Moreover, treatment did not induce any significant changes in concentration of all the major bile acids in the meconium (fig 2 ). With regard to bile acid proportions in the meconium, cholic acid was the predominant species in controls and remained as such in both untreated and UDCA treated ICP groups; chenodeoxycholic acid represented about 30\% of total bile acids and diminished to less than $20 \%$ as a consequence of the disease (fig 2). Despite the significantly lower cholic acid percentage in the maternal serum after UDCA treatment $(23.3$ (3.4) v $61.3(4.9) \%, \mathrm{p}<0.01)$, there was a slightly higher proportion of this bile acid in the meconium (figs 1 and 2). The discrepancy is disclosed by a negative correla- tion for this variable between the two biological fluids $(r=-0.71 ; \mathrm{p}<0.01)$, and furthermore validates the absence of any significant modification in the meconium composition of major bile acids by UDCA administration. Although UDCA percentage in the meconium was slightly increased after treatment compared with the untreated group $(5.2$ (1.2) $v$ $3.9(1.3) \%)$, it was still much lower than that detected in the maternal serum $(28.0(2.8) \%$, $\mathrm{p}<0.01)$. Also, UDCA concentration in the meconium showed only a 2 -fold increase after treatment, despite the much greater increase observed in the maternal serum $(p<0.01)$. Finally, UDCA concentration in the meconium after treatment correlated significantly with the maternal serum concentration of this bile acid $(r=0.74 ; \mathrm{p}<0.01)$, in contrast with that found for the rest of the major bile acids.

Table 2 gives the concentrations of total and individual unusual bile acids in meconium. The data show a significant increase in total unusual bile acids during ICP as compared with the controls. Hyocholic and allo-cholic acids represented the bulk of the unusual bile acid species in normal meconium, accounting for 16.7 (2.7) and $4.1(0.5) \%$ of the total respectively; while allo-cholic acid percentage remained unchanged during ICP, hyocholic acid was considerably decreased by the disease (6.5 (0.8)\%, p<0.01). Also, 12 $\alpha$-hydroxy-3-

Table 2 Usual and unusual bile acids in meconium of newborns from patients with intrahepatic cholestasis of pregnancy

\begin{tabular}{|c|c|c|c|}
\hline & Control & Non-UDCA & $U D C A$ \\
\hline \multicolumn{4}{|l|}{ Usual bile acids $(\mu \mathrm{mol} / \mathrm{g})$} \\
\hline Total & $1.13(0.34)$ & $9.96(4.37)^{\star}$ & $7.17(2.11) \dagger$ \\
\hline \multicolumn{4}{|l|}{ Unusual bile acids $(\mu \mathrm{mol} / \mathrm{g})$} \\
\hline $3 \beta, 7 \alpha$-Dihydroxy- $5 \beta$-cholanoic & $0.03(0.01)$ & $0.19(0.06)^{\star}$ & $0.12(0.04) \dagger$ \\
\hline $3 \alpha, 6 \alpha$-Dihydroxy- $5 \beta$-cholanoic (hyodeoxycholic) & $0.04(0.01)$ & $0.22(0.06)^{\star}$ & $0.15(0.04) \dagger$ \\
\hline $12 \alpha$-Hydroxy-3-oxo- $5 \beta$-cholanoic & $0.02(0.01)$ & $0.35(0.13)^{\star}$ & $0.18(0.04)+\dagger$ \\
\hline $3 \alpha, 7 \alpha, 12 \alpha$-Trihydroxy- $5 \beta$-homocholanoic & $0.01(0.01)$ & $0.07(0.02)^{\star}$ & $0.05(0.01)++\dagger$ \\
\hline $3 \alpha, 6 \alpha, 7 \alpha$-Trihydroxy- $5 \beta$-cholanoic (hyocholic) & $0.30(0.06)$ & $0.62(0.18)$ & $0.51(0.21)$ \\
\hline $3 \alpha, 7 \alpha, 12 \alpha$-Trihydroxy- $5 \alpha$-cholanoic (allo-cholic) & $0.09(0.03)$ & $0.59(0.24)$ & $0.29(0.10)$ \\
\hline $7 \alpha, 12 \alpha$-Dihydroxy-3-oxo-4-cholenoic & $0.03(0.02)$ & $0.16(0.06)$ & $0.06(0.01)$ \\
\hline $3 \alpha$-Hydroxy-7-oxo- $5 \beta$-cholanoic & $0.02(0.01)$ & $0.14(0.04)^{\star}$ & $0.05(0.01) \dagger$ \\
\hline $12 \alpha$-Hydroxy-3,5,7-choltrienoic & $0.01(0.01)$ & $0.09(0.03)^{\star}$ & $0.03(0.01)$ \\
\hline $2 \beta, 3 \alpha, 7 \alpha, 12 \alpha$-Tetrahydroxy- $5 \beta$-cholanoic & $0.02(0.01)$ & $0.08(0.02)$ & $0.03(0.01)$ \\
\hline $3 \alpha, 12 \alpha$-Dihydroxy-7-oxo- $5 \beta$-cholanoic & $0.04(0.02)$ & $0.16(0.04)^{\star}$ & $0.13(0.05)$ \\
\hline Others & $0.25(0.01)$ & $0.84(0.29)^{\star}$ & $0.44(0.10)$ \\
\hline Total & $0.86(0.21)$ & $3.52(1.24)^{\star}$ & $2.04(0.57) \dagger$ \\
\hline Proportion of unusual bile acids & $45.9(3.4)$ & $32.2(5.0)$ & $27.2(6.1) \dagger$ \\
\hline
\end{tabular}

Concentrations of total usual and individual unusual bile acids, and proportion of unusual species in meconium of newborns from normal pregnancies (control; $n=8$ ), and from pregnancies complicated with intrahepatic cholestasis of pregnancy receiving classic palliative therapy (non-UDCA group; $n=7$ ) or treated with ursodeoxycholic acid (UDCA) until labour (UDCA group; $n=9$ ). Values are expressed as mean $(\mathrm{SEM}) .{ }^{\star} \mathrm{p}<0.05$, non-UDCA $v$ control; $\nmid \mathrm{p}<0.05$ and $+\nmid \mathrm{p}<0.01$, UDCA $v$ control. 
Table 3 Pattern of bile acid conjugation in meconium of newborns from patients with intrahepatic cholestasis of pregnancy

\begin{tabular}{lccc}
\hline & Control & Non-UDCA & UDCA \\
\hline Unconjugated & & & \\
CA & $2.5(0.9)$ & $1.7(0.5)$ & $0.8(0.3)$ \\
CDCA & $2.4(0.9)$ & $0.8(0.4)$ & $0.3(0.2) \dagger$ \\
DCA & 0 & $0.7(0.4)$ & $0.3(0.2)$ \\
LCA & 0 & 0 & $0.1(0.1)$ \\
UDCA & 0 & $0.2(0.1)^{\star}$ & $0.3(0.2)$ \\
Glycine conjugated & $15.4(2.9)$ & $33.0(10.4)$ & $36.8(3.2) \dagger \dagger$ \\
CA & $6.7(0.8)$ & $4.1(1.6)$ & $2.4(0.4) \dagger \dagger$ \\
CDCA & $2.2(1.7)$ & $1.5(0.5)$ & $1.1(0.1)$ \\
DCA & $0.2(0.2)$ & $0.6(0.3)$ & $0.4(0.1)$ \\
LCA & $0.2(0.2)$ & $1.0(0.5)$ & $1.2(0.3) \dagger$ \\
UDCA & & & \\
Taurine conjugated & $25.1(8.7)$ & $19.3(7.4)$ & $36.7(8.4)$ \\
CA & $13.5(2.1)$ & $13.6(11.1)$ & $5.0(0.3) \dagger \dagger$ \\
CDCA & $3.2(1.4)$ & $1.4(1.2)$ & $0.8(0.2)$ \\
DCA & $1.3(0.5)$ & $0.2(0.1)$ & $0.2(0.1)$ \\
LCA & $2.1(0.7)$ & $0.4(0.3)^{\star}$ & $0.6(0.1) \dagger$ \\
UDCA & & & \\
Sulphated & $3.0(1.4)$ & $6.7(4.6)$ & $4.0(1.1)$ \\
CA & $21.9(9.4)$ & $12.1(6.3)$ & $4.5(2.4)$ \\
CDCA & $0.2(0.2)$ & $1.5(0.4)^{\star}$ & $0.8(0.7)$ \\
DCA & 0 & $0.6(0.1)^{\star \star}$ & $0.4(0.4)$ \\
LCA & 0 & $0.5(0.1)^{\star \star}$ & $3.5(2.6)$ \\
UDCA & & &
\end{tabular}

Proportions of usual bile acids in meconium from normal pregnancies (control; $n=3$ ), and from pregnancies complicated with intrahepatic cholestasis of pregnancy receiving classical palliative therapy (non-UDCA group) or treated with ursodeoxycholic acid (UDCA) until labour (UDCA group). Meconium samples from newborns of ICP patients included three from each group (low, medium and high total bile acid concentrations). Values are expressed as mean (SEM). ${ }^{\star} \mathrm{p}<0.05$ and ${ }^{\star \star} \mathrm{p}<0.01$, non-UDCA $v$ control; $\nmid \mathrm{p}<0.05$ and $+\dagger \mathrm{p}<0.01$, UDCA $v$ control.

CA, cholic acid; CDCA, chenodeoxycholic acid; DCA, deoxycholic acid; LCA, lithocholic acid.

oxo- $5 \beta$-cholanoic acid was increased both in concentration and percentage as a consequence of ICP ( $\mathrm{p}<0.05)$. UDCA treatment did not influence the bile acid pattern in the meconium of newborns of patients with ICP. Nevertheless, a slight decrease was observed for the concentration and percentage of unusual bile acids after UDCA administration.

After separation of unconjugated and conjugated bile acids in the meconium of newborns from patients with ICP by lipophilic anion exchange chromatography, the unconjugated fraction contained only small proportions $(<3 \%)$ of the total bile acids, irrespective of whether UDCA was given, which was similar to that found in controls ( $5 \%)$ (table 3$)$. As expected, conjugation occurred mainly with taurine $(\sim 45 \%)$, followed by glycine $(\sim 25 \%)$ and sulphate $(\sim 25 \%)$ groups in meconium samples of newborns from healthy pregnant women. Conversely, in meconium during ICP, an equal representation of glycine and taurine conjugates was noticed in both the non-UDCA $(\sim 40$ and $\sim 35 \%$ respectively) and UDCA $(\sim 41$ and $\sim 43 \%$ respectively) groups. The proportion of sulphate conjugated bile acids in meconium of newborns from patients with ICP $(\sim 21 \%)$ was similar to controls, but was smaller in the UDCA group $(\sim 13 \%)$. Sulphated chenodeoxycholic acid was the major species in the sulphate fraction accounting for $\sim 12 \%$ of the total bile acids identified in meconium during ICP, and for $~ 5 \%$ after UDCA treatment.

\section{Discussion}

Data on meconium bile acid composition in newborns from patients with ICP are scant, and changes that occur on UDCA administration during pregnancy have never been evaluated. In this study, we investigated the bile acid profile and the conjugation pattern in meco- nium collected from neonates born of healthy women and of patients with ICP. Serum bile acid composition was also analysed at both diagnosis and delivery to determine whether the bile acid concentration in the maternal serum influences the distribution of these compounds in the meconium. Furthermore, as UDCA has been confirmed as the only effective drug for the treatment of ICP during the last trimester and until delivery, we questioned whether this bile acid reverses alterations in the maternal serum and meconium that the disease may cause. Finally, because concerns have been raised about the toxicity of lithocholic acid or other metabolites of UDCA, we also quantified these species in the meconium.

Maternal intrahepatic cholestasis results in considerable increases in both total bile acid (6-fold) and cholic acid (12-fold) concentration in the serum. Similar alterations in meconium samples of newborns of patients with ICP suggest that these are the result of placental transfer of bile acids from mother to fetus. As the fetal colon is sterile, the presence of products of bacterial metabolism such as deoxycholic and lithocholic acids in increased levels provides further support for the hypothesis that some of the bile acids found in the meconium of these neonates are of maternal origin and reach the fetus by transfer across the placenta. $^{25} 45$

Improvement of pruritus and cholestasis in the mother, ${ }^{13-21}$ as well as a consequent decrease in the bile acid pool in the fetus, have been described as beneficial effects of UDCA treatment. Increasing evidence suggests that UDCA reduces bile acid levels in colostrum, ${ }^{46}$ serum cord blood, ${ }^{22}$ and amniotic fluid. ${ }^{23}$ Interestingly, UDCA administration appears not to influence concentrations of the major bile acids in meconium including that of its toxic metabolite, lithocholic acid, despite the small increase in its percentage detected in maternal serum $(p<0.05)$. UDCA concentration in the meconium is determined by its increase in the maternal serum, but exhibited only a 2-fold increase after treatment, whereas a larger increase was observed in maternal serum. Most striking was the observation that cholic acid percentage significantly decreased in the mother but slightly increased in the fetus. This suggests an accumulation of cholic acid in the meconium, which is retained in the fetal compartment and consequently cannot be modified by treatment. Aggravating this situation is the continued ingestion by the fetus of amniotic fluid which is also enriched in cholic acid. ${ }^{911}$ The lack of effect of UDCA treatment on bile acid concentration in the meconium is not in disagreement with the improved placental transport of bile acids ${ }^{12}$ or the decreased concentrations of total bile acids and cholic acid found in other fetal biological fluids $^{22} 23$ after UDCA administration. On the contrary, the lack of ability of the fetus to excrete meconium (in normal conditions) and the reduced intestinal absorption are responsible for accumulation of bile acids in the meconium which cannot be modified by the 
treatment. In this sense, UDCA treatment should be initiated as soon as the diagnosis of ICP is made, mainly to decrease bile acid levels in the maternal serum and therefore restrict the accumulation of these compounds in the meconium.

As bile acid metabolism in the newborn infant differs from that in the adult, it is not unexpected that a variety of unusual bile acids should be present in normal meconium, resembling the composition of fetal gallbladder bile. $^{2627}$ In addition to cholic, chenodeoxycholic, deoxycholic, and lithocholic acids, significant amounts of hyocholic, hyodeoxycholic, allo-cholic, and $7 \alpha, 12 \alpha-$ dihydroxy-3-oxo-4-cholenoic acids were also identified during this work, which is in accordance with the results of others. ${ }^{24} 2847-49$ Their increased levels in meconium during ICP are probably due to the ingestion of amniotic fluid containing increased amounts of bile acids as a consequence of cholestasis. Moreover, the presence of unusual bile acids may also reflect hepatic immaturity and/or activation of liver enzymes that are not important later in life.

In agreement with our data, previous studies have shown that $6 \alpha$-hydroxylation of bile acids is a major metabolic reaction during the prenatal period ${ }^{24}{ }^{50}$ and therefore hyocholic acid is the predominant unusual bile acid found in meconium. In this study, proportions of hyocholic (17\%) and chenodeoxycholic (31\%) acids in normal meconium were similar to those (19\% and $29 \%$ respectively) reported previously. ${ }^{28}{ }^{47}$ Interestingly, the significant decrease in chenodeoxycholic acid percentage in meconium during ICP was paralleled by a similar decrease in hyocholic acid proportion. These changes were not influenced by UDCA treatment.

Although bile acid conjugation in normal meconium was more pronounced with taurine than with glycine, in agreement with the results of others, ${ }^{50} 51$ the predominance of glycine conjugates during ICP found in this study may result from restricted maternal to fetus transport across the placenta for taurine conjugates. With regard to the sulphate conjugated fraction, it has been shown to represent a large proportion of the total bile acids in meconium of newborns from both uncomplicated pregnancies and ICP. ${ }^{52}$ These findings were confirmed in this study, in which sulphated bile acids accounted for $21 \%$ of the total in meconium of neonates from untreated ICP, but were only $\sim 13 \%$ in neonates from UDCA treated ICP. Whether a decrease in sulphation, considered by some to be an important pathway to the detoxification of hepatotoxic bile acids, is due to the effect of UDCA in the fetus is still to be investigated.

In conclusion, increased bile acid levels in maternal serum during ICP induce a considerable increase in these compounds in the meconium, with cholic acid representing the major species. UDCA administered to the mother until labour corrects the bile acid profile in the maternal serum but does not influence the meconium bile acid pattern of ICP. Moreover, this beneficial treatment for the mother does not increase meconium levels of potentially toxic metabolites of UDCA such as lithocholic acid.

We thank attending physicians from the Santa Maria Hospital, Garcia de Orta Hospital and Dr Alfredo da Costa Maternity Garcia de Orta Hospital and Dr Alfredo da Costa Maternity
Ward, Portugal for their help with recruiting the patients, and Dr Falk Pharma, GmbH, Germany for financial support for part Dr Falk Phar
of this work.

1 Reyes H. The enigma of intrahepatic cholestasis of pregnancy: lessons from Chile. Hepatology 1982;2:87-96.

2 Van Dyke RW. The liver in pregnancy. In: Zakim D, Boyer TD, eds. Hepatology: a textbook of liver disease. Philadelphia: WB Saunders, 1990;2:1438-59.

3 Reyes H, Gonzalez MC, Ribalta J, et al. Prevalence of intrahepatic cholestasis of pregnancy in Chile. Ann Intern Med 1978;88:487-93.

4 Brites D, Rodrigues CMP, van-Zeller $\mathrm{H}$, et al. Relevance of serum bile acid profile in the diagnosis of intrahepatic cholestasis of pregnancy in a high incidence area: Portugal. cholestasis of pregnancy in a high incidence are:

5 Fisk NM, Storey GNB. Fetal outcome in obstetric cholestasis. Br F Obstet Gynaecol 1988;95:1137-43.

6 Reyes H, Radrigán ME, Gonzalez MC, et al. Steatorrhea in patients with intrahepatic cholestasis of pregnancy. Gastroenterology 1987;93:584-90.

7 Alsulyman OM, Ouzounian JG, Ames-Castro M, et al. Intrahepatic cholestasis of pregnancy: perinatal outcome associated with expectant management. Am $\mathcal{F}$ Obstet Gynecol 1996;175:957-60.

8 Laatikainen T. Fetal bile acid levels in pregnancies complicated by maternal intrahepatic cholestasis. Am $\mathcal{f}$ Obstet Gynecol 1975;122:852-6.

9 Heikkinen J, Mäentausta O, Tuimala R, et al. Amniotic fluid bile acids in normal and pathologic pregnancy. Obstet Gynecol 1980;56:60-4.

10 Shaw D, Frohlich J, Wittmann BAK, et al. A prospective study of 18 patients with cholestasis of pregnancy. $A m \mathcal{F}$ Obstet Gynecol 1982;142:621-5.

11 Heikkinen J, Ylöstalo P, Mäentausta O, et al. Bile acids in maternal serum, umbilical cord serum and amniotic fluid of healthy women, women with pruritus and patients with intrahepatic cholestasis of pregnancy. I Obstet Gynaecol $1983 ; 4: 17-20$

12 Serrano MA, Brites D, Larena MG, et al. Beneficial effect of ursodeoxycholic acid on alterations induced by cholestasis of pregnancy in bile acid transport across the human placenta. F Hepatol 1998;28:829-39.

13 Mazzella G, Rizzo N, Salzetta A, et al. Management of intrahepatic cholestasis in pregnancy. Lancet 1991;338:1594-

14 Palma J, Reyes H, Ribalta J, et al. Effects of ursodeoxycholic acid in patients with intrahepatic cholestasis of pregnancy. Hepatology 1992;15:1043-7.

15 Floreani A, Paternoster D, Grella V, et al. Ursodeoxycholic acid in intrahepatic cholestasis of pregnancy. Br $\mathcal{f}$ Obstet Gynaecol 1994;101:64-5.

16 Davies MH, da Silva RCMA, Jones SR, et al. Fetal mortality associated with cholestasis of pregnancy and the potential associated with cholestasis of pregnancy and the potential $580-4$.

17 Meng, L-J, Reyes H, Axelson M, et al. Progesterone metabolites and bile acids in serum of patients with intrahepatic cholestasis of pregnancy: effect of ursodeoxycholic acid therapy. Hepatology 1997;26:1573-9.

18 Meng, L-J, Reyes H, Palma J, et al. Effects of ursodeoxycholic acid on conjugated bile acids and progesterone metabolites in serum and urine of patients with intrahepatic cholestasis of pregnancy. $\mathcal{F}$ Hepatol 1997;27: 1029-40.

19 Palma J, Reyes H, Ribalta J, et al. Ursodeoxycholic acid in the treatment of cholestasis of pregnancy: a randomized, double-blind study controlled with placebo. If Hepatol 1997;27:1022-8.

20 Brites D, Rodrigues CMP, Oliveira N, et al. Correction of maternal serum bile acid profile during ursodeoxycholic acid therapy in cholestasis of pregnancy. F Hepatol 1998;28: acid the $91-8$.

21 Brites D, Rodrigues CMP, Cardoso MC, et al. Unusual case of severe cholestasis of pregnancy with early onset, improved by ursodeoxycholic acid administration. Eur $\mathcal{f}$ Obstet Gynecol Reprod Biol 1998;76:165-8.

22 Brites $\mathrm{D}$, Oliveira $\mathrm{N}$, van-Zeller $\mathrm{H}$, et al. Correction of fetal cord serum bile acid profile by maternal ursodeoxycholic acid therapy in cholestasis of pregnancy [abstract]. $\mathcal{F}$ Hepatol 1997;26:163A

23 Brites D, El-Mir MY, Oliveira N, et al. Amniotic fluid bile acid changes in the course of ursodeoxycholic acid therapy in intrahepatic cholestasis of pregnancy [abstract]. 7 Hepatol 1997;26:164A.

24 Back P, Walter K. Developmental pattern of bile acid metabolism as revealed by bile acid analysis of meconium. Gastroenterology 1980;78:671-6.

25 Lester R, Pyrek Jst, Little JM, et al. Nature of bile acids in the fetus and newborn infant. $₹$ Pediatr Gastroenterol Nutr the fetus and new

26 Colombo C, Zuliani G, Ronchi M, et al. Biliary bile acid composition of the human fetus in the early gestation. Pediatr Res 1987;21:197-200. 
27 Setchell KD, Dumaswala R, Colombo C, et al. Hepatic bile acid metabolism during early development revealed from 1988;263:16637-44.

28 Kimura A, Yamakawa R, Ushijima K, et al. Fetal bile acid metabolism during infancy: analysis of $1 \beta$-hydroxylated bile acids in urine, meconium and feces. Hepatology 1994;20:819-24

29 Labbé D, Gerhard M-F, Myara A, et al. High-performance liquid chromatographic determination of tauro- and glycoconjugated bile acids in human serum. F Chromatogr 1989 490:275-84.

30 Feldmann D, Fenech C, Cuer JF. Evaluation of a sample preparation procedure for bile acids in serum and bile. Clin Chem 1983;29:1694.

31 Mashige F, Imai K, Osuga T. A simple and sensitive assay of bile acids. Clin Chim Acta 1976;70:79-86.

32 Brites D, Silva R. Doseamento enzimático dos ácidos biliares. Optimização da reacção e valores normais. Revista Portuguesa de Farmácia 1985;36:8-14.

33 Beke R, De Weerdt GA, Parijs J, Huybrechts et al. Separation of conjugated and unconjugated bile acids by Separation of conjugated and unconjugated bile acids by
thin layer chromatography. Clin Chim Acta 1976;70:197-9

34 Goswami SK, Frey CF. Thin-layer chromatographic method for the separation of conjugated deoxycholic acid from conjugated chenodeoxycholic acid. $\mathcal{F}$ Chromatogr 1978; 145:147-50

35 Correia LE, Brites D. Separação, identificação e quantificação dos diferentes ácidos biliares no soro. Revista Portuguesa de Farmácia 1985;36:30-5.

36 Setchell KDR, Lawson AM, Tanida N, et al. General methods for the analysis of metabolic profiles of bile acids and related compounds in feces. F Lipid Res 1983;24:1085-100.

37 Hirano Y, Miyazaki $\mathrm{H}$, Higashidate S, et al Analysis of 3-sulfated and nonsulfated bile acids by one-step hydrolysis and high performance liquid chromatography. $\mathcal{F}$ Lipid Res 1987;28:1524-9.

38 Nair PP, Garcia CC. A modified gas-liquid chromatographic procedure for the rapid determination of bile acids in biological fluids. Anal Biochem 1969;29:164-6.

39 Almé B, Bremmelgaard A, Sjövall J, et al. Analysis of metabolic profiles of bile acids in urine using a lipophilic anion exchanger and computerized gas-liquid chromatographymass spectrometry. $\mathscr{F}$ Lipid Res 1977;18:339-62.
40 Fales HM, Jaouni JM, Babashak JF. Simple device for preparing ethereal diazomethane without resorting to
co-distillation. Anal Chem 1973;45:2302-3.

41 Blau K, King GS. Handbook of derivatives for chromatography. London: Heyden \& Son, 1978.

42 Axelson M, Sjövall J. Separation and computerized gas chromatography-mass spectrometry of unconjugated neutral steroids in plasma. $\mathcal{F}$ Steroid Biochem 1974;5:733-8.

43 Lawson AM, Setchell KDR. Mass spectrometry in bile acids. In: Setchell KDR, Kritchevsky D, Nair P, eds. The bile acids. New York: Plenum Press, 1988;4:167-267.

44 Setchell KDR. Liquid-solid extraction, lipophilic gel chromatography and capillary column gas chromatography in
the analysis of bile acids from biological samples. In: Barbara L, Dowling RH, Hofmann AF, et al, eds. Bile acids in gastroenterology. Lancaster: MTP Press, 1982:1-18.

45 Sewell RB, Hardy KJ, Smallwood RA, et al. Fetal bile salt metabolism: placental transfer of dihydroxy bile salts in sheep. Am F Physiol 1982;243:G172-5.

46 Brites D, Rodrigues CMP. Elevated levels of bile acids in colostrum in patients with cholestasis of pregnancy are decreased following ursodeoxycholic acid therapy. $\mathcal{F}$ Hepatol 1998;29:743-51.

47 Kurosawa T, Mahara R, Nittono $\mathrm{H}$, et al. Synthesis of 6-hydroxylated bile acids and identification of $3 \alpha, 6 \alpha, 7 \alpha, 12 \alpha$-tetrahydroxy- $5 \beta$-cholan-24-oic acid in human meconium acid in (Tokyo) 1989;37:557-9.

48 Inoue T, Kimura A, Aoki K, et al. Developmental pattern of 3-oxo- $\Delta^{4}$ bile acids in neonatal bile acid metabolism. Arch Dis Child Fetal Neonatal Ed 1997;77:F52-6.

49 Murai T, Mahara R, Kurosawa T, et al. Determination of fetal bile acids in biological fluids from neonates by gas chromatography-negative ion chemical ionization mass spectrometry. F Chromatogr B Biomed Appl 1997;28:13-22.

50 Jönsson G, Midtvedt A-C, Norman A, et al. Intestinal microbial bile acid transformation in healthy infants. $\mathcal{F}$ Pediatr Gastroenterol Nutr 1995;20:394-402.

51 Sharp HL, Peller J, Carey JB. Primary and secondary bile acids in meconium. Pediatr Res 1971;5:274-9.

52 Laatikainen TJ, Lehtonen PJ, Hesso AE. Fetal sulfated and nonsulfated bile acids in intrahepatic cholestasis of pregnancy. F Lab Clin Med 1978;92:185-93. 